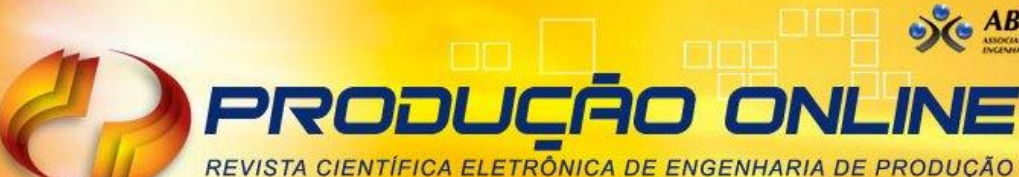 \\ REVISTA CIENTIFICA ELETRÔNICA DE ENGENHARIA DE PRODUÇÃO \\ ISSN 1676-190
}

\section{GRASP DUPLAMENTE REATIVA COM PATH-RELINKING PARA O PROBLEMA DE P-MEDIANAS APLICADO À FORMAÇÃO DE CLUSTERS EM LOGÍSTICA DE LOCALIZAÇÃO}

\section{DOUBLY REACTIVE GRASP WITH PATH-RELINKING TO P-MEDIAN PROBLEM APPLIED TO CLUSTERS FORMATION IN LOCATION LOGISTICS}

Caroline Nascimento Parajara* E-mail: carolparajara@hotmail.com

Geraldo Galdino de Paula Junior* E-mail: galdino@uenf.br

*Universidade Estadual do Norte Fluminense (UENF), Campos dos Goytacazes, RJ, Brasil

Resumo: Este trabalho relata a experiência de formular, construir e testar um procedimento duplamente reativo para a meta-heurística GRASP com path-relinking destinado à solução do problema generalizado de $p$-medianas aplicado à logística de localização. São usados dois parâmetros de reação para controlar a busca de soluções na fase de construção da GRASP. O uso simultâneo dos dois parâmetros reativos permitiu criar uma disciplina para alocação dos clientes aos agrupamentos das medianas: clientes mais próximos são alocados primeiro. Para implementação do path-relinking, um conjunto elite de soluções foi criado através de um conceito denominado "índice vocação mediana" que estabelece o quanto cada cidade está próxima das demais cidades do grupo. Para testar a qualidade das soluções, foi utilizado um problema envolvendo as cidades brasileiras de médio e grande porte.

Palavras-chave: Problema de localização. GRASP com path-relinking e índice vocação mediana.

Abstract: This paper reports the experience in formulating, building and testing a procedure for a doubly reactive GRASP with path-relinking for the solution of the general problem of $p$-median applied to location logistic. Two reaction parameters were used to control the search for solutions in the construction phase of the GRASP. The simultaneous use of two reactive parameters allowed creating a disciplined allocation of clients to groups of medians: customers closer are allocated first. To implement the path-relinking, a set of elite solutions was created through a concept called "median vocation index" that establishes how much each city is close to the other cities of the group. To test the quality of solutions, a problem involving medium and large Brazilian cities was employed.

Keywords: Location problem. GRASP with path-relinking and median vocation index.

\section{INTRODUÇÃO}

Localização de instalações tem sido assunto de interesse da Engenharia de Produção, Engenharia de Transporte, de administradores, de executivos ligados à logística, etc. O problema pode ser abordado de forma qualitativa ou quantitativa, ou ainda adotando uma abordagem híbrida dos dois enfoques.

Problemas de localização associam-se a um conjunto de instalações que minimizam o custo de satisfazer a demandas e obedecem a um elenco de restrições. 
Modelos e decisões locacionais são de natureza econômica e visam organizar 0 atendimento eficiente a algum tipo de demanda. Modelos locacionais são usados em aplicações que têm como meta e ênfase otimizar o custo ou tempo de atendimento, entre outros itens de interesse.

Especializando o tema para a indústria e a distribuição de seus produtos, o que se procura determinar é "onde" tais facilidades devem ser localizadas, "quais" fornecedores devem ser utilizados e com "quantos" centros de distribuição a empresa deve operar. Estudos que antecedem e sucedem os projetos locacionais procuram determinar o tipo de cliente ou de zonas de mercado que devem ser supridos a partir de cada centro de distribuição.

Em geral, não se pode garantir que problemas de localização sejam resolvidos de maneira exata por algoritmos em tempo polinomial determinístico. Portanto, suas soluções podem ficar comprometidas quanto a velocidade de emissão dos resultados. Este fato motiva a utilização de algoritmos heurísticos para proverem soluções viáveis tão boas quanto possíveis, eventualmente ótimas, para os problemas.

A meta-heurística GRASP (Greedy Randomized Adaptive Search Procedure) (ver em Feo \& Resende (1995)) vem produzindo excelentes resultados na solução de problemas combinatórios importantes. Variações da GRASP como RGRASP (GRASP REATIVA), conforme García (2001), e GRASP com religamento de caminhos (path-relinking), de acordo com Resende \& Werneck (2002a), Boudia et al. (2007), Caires et al. (2010) e Frinhani (2011) têm produzido resultados interessantes neste contexto.

\section{GRASP}

Proposta por Feo \& Resende (1995), a meta-heurística GRASP (Greedy Randomized Adaptive Search Procedure) é um método iterativo que consiste de duas fases: uma fase de construção, na qual uma solução gulosa e aleatória é gerada, elemento a elemento; e uma fase de busca local, na qual um ótimo local na vizinhança da solução construída é pesquisado. A melhor solução encontrada ao longo de todas as iterações realizadas é retornada como resultado da GRASP.

A seguir, a GRASP é apresentada através do Algoritmo 1. 


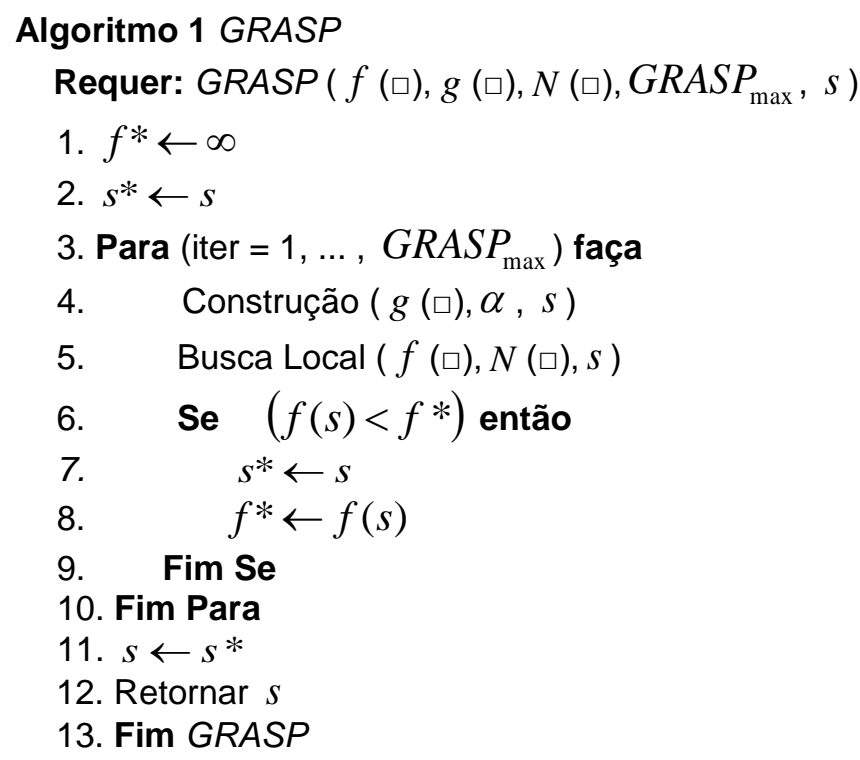

\subsection{Fase de construção da GRASP}

A fase de construção da GRASP começa com uma solução viável sendo construída iterativamente, elemento por elemento. A cada iteração, os próximos elementos a serem incluídos na solução são colocados em uma lista $C$ de candidatos seguindo um critério de ordenação pré-determinado. A seleção é baseada em uma função gulosa $g: C \rightarrow \Re$, que mede o benefício da seleção de cada candidato a compor a solução. A componente probabilística da GRASP vem do fato de que cada elemento é selecionado de forma aleatória a partir de uma lista que contém os melhores elementos que compõem a lista de candidatos, chamada lista restrita de candidatos $(L R C)$. Esta técnica de escolha permite que em cada iteração diferentes soluções sejam geradas.

O pseudocódigo da Fase de Construção é apresentado pelo algoritmo 2.

O tamanho da $L R C$ é controlado por um parâmetro $\alpha$, onde $0 \leq \alpha \leq 1$. ○ $\alpha$ pode ser, então, considerado como o principal parâmetro da GRASP, pois a qualidade das soluções depende de seu ajuste 


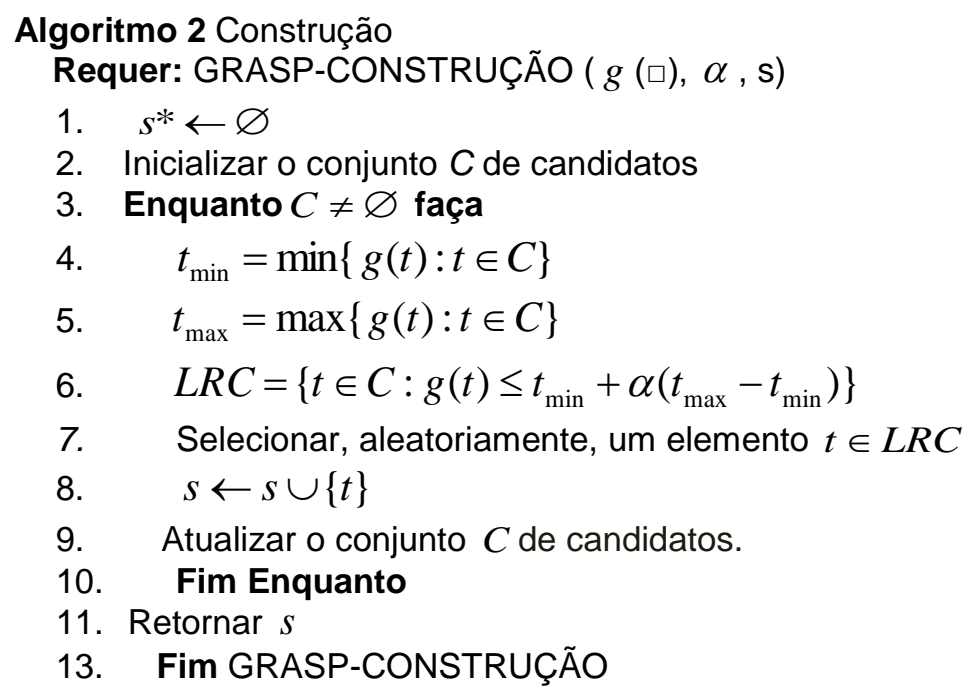

\subsection{Cálculo da função gulosa da GRASP para p-medianas}

Neste trabalho, a função gulosa necessária na fase de construção da GRASP, relativa à $i$-ésima mediana, $i \leq p$, é definida por

$$
g_{i}\left(C_{i}\right)=g_{\text {min }}^{i}+\alpha\left(g_{\text {max }}^{i}-g_{\text {min }}^{i}\right)
$$

onde $g_{\min }^{i}$ é escolhido de tal maneira que $g_{\min }^{i}<g_{\max }^{i}$ e

$$
g_{\max }^{i}\left(C_{i}\right)=\frac{\sum_{j \in C_{i}} \Delta_{i j}}{\left|C_{i}\right|-1}, \quad\left|C_{i}\right|>1
$$

ou

$$
g_{\text {max }}^{i}\left(C_{i}\right)=\frac{f_{i}+\sum_{j \in C_{i}} \Delta_{i j}}{\left|C_{i}\right|-1}, \quad\left|C_{i}\right|>1
$$

Tanto na função (1) quanto nas funções (2) e (3), $C_{i}$ representa um possível agrupamento da mediana $i \cdot \Delta_{i j}$ é a diferença entre a distância da mediana $i$ à cidade de menor distância, e a distância da mediana $i$ à cidade $j$. No caso da função (3), $f_{i}$ é o custo fixo da mediana $i$. Em qualquer dos dois casos, o agrupamento (com sua mediana) será tão melhor quanto menor for a média dos custos representada pelas funções.

Nas fórmulas (1) e (2), o termo -1 no denominador se refere ao nó (cidade) mais próxima da mediana, que não participa do somatório. Para ela - cidade mais próxima - a distância adicional, em relação a distância mínima, é zero $\left(\Delta_{i j}=0\right)$. 
Para ilustrar o uso da função gulosa, considera-se uma matriz de distâncias para 10 cidades, de acordo com a Tabela 1. Aleatoriamente a cidade 8 é escolhida candidata a mediana.

A cidade mais próxima é a 3 que está a uma distância 5 da cidade 8 . As demais cidades para serem atendidas por 8, percorrerão esses 5 mais uma distância adicional: a cidade 1 está distante 12: 5+7. A cidade 2 está distante 18: 5+13, e assim por diante. A seguir são feitos os outros cálculos com auxílio da Tabela 1.

- $g_{\max }^{8}=\frac{\sum_{j \in C_{8}} \Delta_{8 j}}{\left|C_{8}\right|-1}=\frac{7+13+1+9+4+5+3+35}{8}=\frac{77}{8}=9,625 \rightarrow 10 ;$

- $g_{\min }^{8}=0$;

- $\alpha=0,7$;

- $g^{8}=g_{\min }^{8}+0,7 \times\left(g_{\max }^{8}-g_{\min }^{8}\right)=0,7 \times 10=7$.

Tabela 1- Matriz de distâncias para ilustrar o cálculo da função gulosa, com 10 cidades

\begin{tabular}{ccccccccccc}
\hline $\mathbf{1}$ & $\mathbf{1}$ & $\mathbf{2}$ & $\mathbf{3}$ & $\mathbf{4}$ & $\mathbf{5}$ & $\mathbf{6}$ & $\mathbf{7}$ & $\mathbf{8}$ & $\mathbf{9}$ & $\mathbf{1 0}$ \\
$\mathbf{2}$ & $*$ & $*$ & $*$ & $*$ & $*$ & $*$ & $*$ & $*$ & $*$ & $*$ \\
$\mathbf{3}$ & $*$ & $*$ & $*$ & $*$ & $*$ & $*$ & $*$ & $*$ & $*$ & $*$ \\
$\mathbf{4}$ & $*$ & $*$ & $*$ & 0 & $*$ & $*$ & $*$ & $*$ & $*$ & $*$ \\
$\mathbf{5}$ & $*$ & $*$ & $*$ & $*$ & 0 & $*$ & $*$ & $*$ & $*$ & $*$ \\
$\mathbf{6}$ & $*$ & $*$ & $*$ & $*$ & $*$ & 0 & $*$ & $*$ & $*$ & $*$ \\
$\mathbf{7}$ & $*$ & $*$ & $*$ & $*$ & $*$ & $*$ & 0 & $*$ & $*$ & $*$ \\
$\mathbf{8}$ & 12 & 18 & $\mathbf{5}$ & 6 & 14 & 9 & 10 & 0 & 8 & 40 \\
$\mathbf{9}$ & $5+7$ & $5+13$ & $5+0$ & $5+1$ & $5+9$ & $5+4$ & $5+5$ & mediana & $5+3$ & $5+35$ \\
$\mathbf{1 0}$ & $*$ & $*$ & $*$ & $*$ & $*$ & $*$ & $*$ & $*$ & $*$ & 0 \\
\hline
\end{tabular}

Escolha dos clientes da mediana candidata 8, com $\alpha=0,7$. Em cada linha usa-se $\frac{\sum_{j \in C_{i}} \Delta_{i j}}{\left|C_{i}\right|-1}$

- $\quad \frac{1}{1}=1<7$, escolhe o 4 ;

- $\frac{1+3}{2}=2<7$, escolhe o 9 ;

Revista Produção Online, Florianópolis, SC, v.15, n. 3, p.807-829, jul./set. 2015. 
- $\frac{1+3+4}{3}=2, \ldots<7$, escolhe o 6

- $\frac{1+3+4+5}{4}=3, \ldots<7$, escolhe o 7

- $\frac{1+3+4+5+7}{5}=4<7$, escolhe o 1

- $\frac{1+3+4+5+7+9}{6}=5, \ldots<7$, escolhe o 5 ;

- $\frac{1+3+4+5+7+9+13}{7}=6<7$, escolhe o 2

- $\frac{1+3+4+5+7+9+13+35}{8}=9,625>7$, NÃO escolhe 010 .

Neste caso, o agrupamento da mediana 8 é $\{4,9,6,7,1,5,2\}$.

Escolha dos clientes da mediana candidata 8, com $\alpha=0,3$. Neste caso, $g^{8}=g_{\min }^{8}+0,3 \times\left(g_{\max }^{8}-g_{\min }^{8}\right)=0,3 \times 10=3$.

- $\frac{1}{1}=1<3$, escolhe o 4 ;

- $\frac{1+3}{2}=2<3$, escolhe o 9

- $\frac{1+3+4}{3}=2, \ldots<3$, escolhe o 6

- $\quad \frac{1+3+4+5}{4}=3, \ldots>3, \mathrm{NÃO}$ escolhe 07 .

Aqui, o agrupamento da mediana 8 é $\{4,9,6\}$. Observa-se que quanto menor for $0 \alpha$, mais se restringe a escolha de cidades clientes da mediana candidata.

Os primeiros projetos da GRASP trabalhavam com um $\alpha, 0 \leq \alpha \leq 1$, que era calibrado até se tornar um parâmetro fixo do método. Com a evolução da pesquisa, foram adotados esquemas reativos em que é permitido ao $\alpha$ sofrer variações durante as sucessivas iterações do algoritmo, buscando um valor ideal para ele.

\subsection{GRASP Reativa}

Segundo García (2001), uma das possíveis desvantagens dos algoritmos baseados em meta-heurísticas é a falta de robustez, no sentido de que sua eficácia pode ser muito dependente da escolha de valores dos parâmetros. Isso geralmente Revista Produção Online, Florianópolis, SC, v.15, n. 3, p.807-829, jul./set. 2015. 
implica em uma tarefa considerável para "ajustar" esses valores, a fim de obter maior desempenho. Isto levou ao estudo dos chamados métodos reativos, que, em essência, são algoritmos baseados em meta-heurísticas de auto-ajuste.

\subsubsection{Novo procedimento reativo para a GRASP - a metáfora da peneira}

O procedimento reativo para a GRASP é conduzido provocando uma variação do $\alpha, 0 \leq \alpha \leq 1$, na fase de construção do método.

\section{Variando os orifícios da tela da peneira}

Uma das possibilidades da GRASP reativa consiste em começar com $0 \alpha$ bem pequeno visando com isso escolher/alocar para cada mediana os nós (clientes) mais próximos. Em seguida o $\alpha$ é aumentado progressivamente, permitindo que clientes mais distantes de cada mediana sejam alocados. $\mathrm{O} \alpha$ funcionaria como uma espécie de controlador dos orifícios da tela da peneira que é iniciada com uma tela bem fina. No início, o material peneirado é constituído de grãos muito pequenos - ou finos (que representam os clientes mais próximos a serem alocados). A tela da peneira é progressivamente substituída por outra tela mais grossa, ou seja, com espaços maiores entre os fios da tela. O material peneirado vai se tornando progressivamente granulado com grãos maiores (representando os clientes mais distantes a serem alocados por último). Cria-se com isso uma disciplina de alocação: clientes mais próximo são alocados primeiro.

\section{Variando o tamanho da peneira}

Simultaneamente ao processo de aumentar progressivamente os orifícios da tela da peneira, fazendo o $\alpha$ crescer no intervalo [0,1], o tamanho da peneira, em si, é também controlado de acordo com $g=g_{\text {min }}+\alpha\left(g_{\max }-g_{\text {min }}\right)$, onde

$$
\begin{aligned}
g_{\text {min }} & =g_{\text {min }}\left(\gamma, g_{\text {max }}\right) \\
& =\gamma \cdot g_{\text {max }}, \quad 0 \leq \gamma<1
\end{aligned}
$$

Com a alteração iterativa do parâmetro $\gamma$, o valor da diferença $g_{\max }-g_{\min }$ se altera também de forma iterativa. Cria-se com isso um processo GRASP 2 REATIVA, em que a reação se dá, simultaneamente, pelos dois procedimentos descritos. 


\subsection{A fase de melhoria ou busca local da GRASP}

Existe uma variedade de procedimentos possíveis para conduzir esta fase da meta-heurística. Alguns desses esquemas podem ser vistos em García (2001). Na fase de melhoria das soluções da GRASP, adotada neste trabalho, o algoritmo compara o custo de alocação ao agrupamento original - vindo da fase de construção - de cada cidade cliente com o custo de sua hipotética alocação aos demais agrupamentos. Se outro agrupamento oferece um custo menor de atendimento, ou seja, uma proximidade melhor da mediana, a cidade cliente é retirada do agrupamento em que ela está no momento, e alocada ao agrupamento de menor custo de alocação. Para haver uma troca, a diferença entre o que se ganha e o que se perde com a troca, deve diminuir o custo de alocação do cliente em questão.

Segundo Rangel et al. (2000), a fase de melhoria é necessária pelo fato das soluções iniciais da GRASP não serem necessariamente ótimos locais. O objetivo da fase de melhoria, então, é melhorar a solução criada na fase construtiva. Essa busca por melhores soluções se dá consultando a estrutura de vizinhança, $\operatorname{Viz}(s)$, da solução $s$ construída, através de sucessivas trocas da solução corrente, sempre que uma melhor solução é encontrada. Várias são as trocas: pode-se fechar uma planta e alocar seus clientes à outras plantas; pode-se fechar uma planta e abrir uma que estava fechada; mudar um cliente de uma planta para outra; trocar de planta dois clientes, etc.

A seguir, o algoritmo 3 apresenta o pseudocódigo da fase de melhoria ou busca local. 


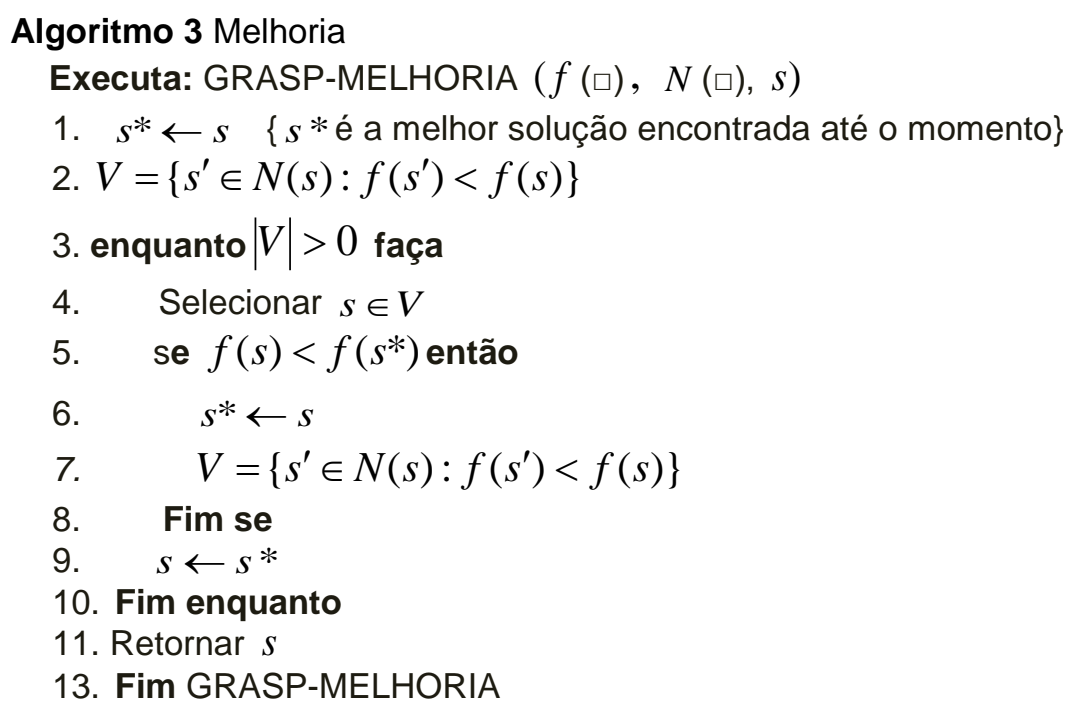

\subsection{GRASP modificada: inclusão dos parâmetros de reação}

Os algoritmos 4 e 5, que são os algoritmos 1 e 2 alterados, incorporam as modificações que tornam a GRASP duplamente reativa. Esta modificação afeta somente a parte inicial do algoritmo e a fase de construção.

$\mathrm{Na}$ implementação o intervalo $[0,1]$ é discretizado para fornecer valores numéricos aos parâmetros de reação. Por exemplo, $0 \leq \alpha \leq 1$ assume os valores do conjunto $\{0,0.1, \ldots, 0.9\}$, no decorrer das iterações. Do mesmo modo, $0 \leq \gamma<1$ assume os valores de $\{0,0.1, \ldots, 0.9\}$.

\section{PATH-RELINKING}

O path-relinking foi sugerido como enfoque para integrar estratégias de intensificação e diversificação num esquema de busca. $O$ enfoque pode ser visto como uma instância - altamente focada - de uma estratégia que procura incorporar atributos de soluções de alta qualidade (soluções elite), procurando contemplar ou favorecer esses atributos nos movimentos selecionados (GLOVER et al., 2003, apud GLOVER \& KOCHENBERGER, 2003). 

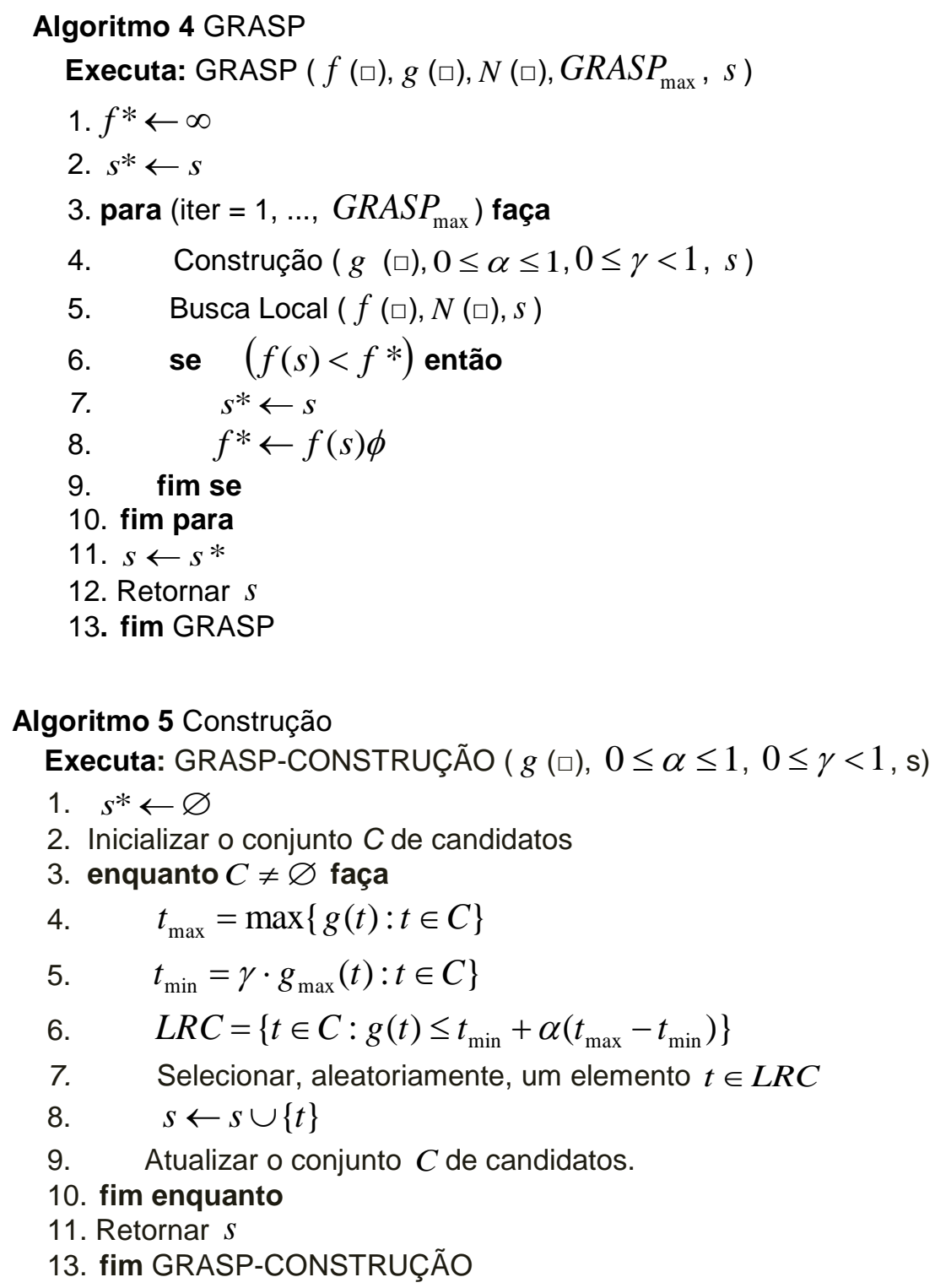

Um procedimento equivalente seria construir caminhos usando uma combinação de várias soluções até que todas fossem examinadas.

O path-relinking se associa à ideia de reinterpretar as combinações lineares de pontos no Espaço Euclidiano como caminhos entre e além das soluções no âmbito da vizinhança. O caminho entre duas soluções no espaço de busca pode produzir soluções que compartilham atributos comuns a soluções previamente existentes. A sequência de soluções vizinhas no espaço de decisão é gerada a partir da solução inicial, indo na direção da solução alvo. A melhor solução encontrada na sequência é retornada.

Intensificação e diversificação em qualquer método de busca devem ser tratadas com a ideia de se manter um equilíbrio entre elas durante o processo. A 
proposta do path-relinking foi feita como um instrumento capaz de garantir, ou pelo menos permitir a integração equilibrada dessas estratégias de intensificação e diversificação.

O path-relinking é um enfoque que pode ser usado na fase terminal de uma meta-heurística "quase como uma pós-otimização, ou pós-melhoria no caso da GRASP" para gerar novas soluções através do exame de trajetórias que ligam soluções de alta qualidade - novas ou já existentes - a partir da solução inicial.

Desse ponto inicial em diante o procedimento explora o espaço de busca gerando um caminho que leva a outras soluções, chamadas de soluções guia. A seleção dos movimentos nesta fase é feita explorando os atributos de soluções já conhecidas, em particular, aqueles das soluções guia. Enfim, o path-relinking pode ser visto como uma estratégia que atua no sentido de incorporar atributos de soluções de alta qualidade, tentando privilegiar esses atributos nos movimentos que são progressivamente selecionados.

A Figura 1 mostra dois caminhos possíveis ligando a solução $x_{1}=$ "início" à solução $x_{n}=$ "alvo", para ilustrar o religamento entre soluções. O caminho original é representado por segmentos de linha interrompida e decorre da "operação normal" de um algoritmo que produz seu resultado na forma de uma série de movimentos que conduzem do ponto $x_{1}$ ao ponto $x_{n}$, isto é, gera a sequência ("início"= $x_{1}, x_{2}, x_{3}, \ldots, x_{n}=$ "alvo") enquanto que o caminho de religamento é representado pelos segmentos de linha sólida.

Os caminhos são gerados com base na seleção de movimentos e adotam uma trajetória em que os passos intermediários são determinados por alguma função de avaliação. A Figura 1 mostra uma solução (o nó escuro associado a $x_{\min }$ ) alcançada pelo caminho de linha sólida. Além disso, o caminho religado pode encontrar soluções possivelmente melhores do que a solução guia inicial - que, nesta ilustração, pode ser $x_{n}$ - ou provê "entradas férteis" para alcançar outras soluções, de alguma forma, melhores. Em geral, as soluções guia podem ser constituídas a partir de uma seleção de soluções anteriores chamadas de "soluções elite". 
Figura 1 - Dois caminhos: o original e o path-relinking - em linhas sólidas

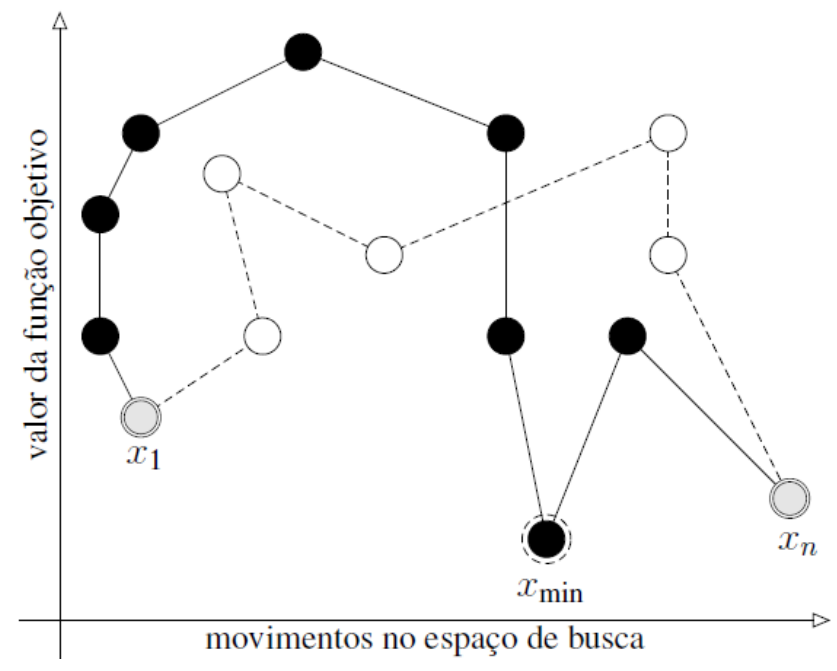

\section{O algoritmo 6 apresenta o pseudocódigo do path-relinking.}

\section{Algoritmo 6 GRASP COM PATH-RELINKING}

Executa: GRASP COM PATH-RELINKING $\left(s_{k}, M A X k_{\text {PATHRELINKING }}\right.$,

$X_{1}, \ldots, X_{\text {MAXk }_{\text {PATHRELNING }}}, Y, S_{\text {PATHRELINKING }}$ )

1. // $X_{K}$ para $k=1, \ldots, M A X k_{\text {PATHRELINKING }}$, contém dados originais do //problema de localização como candidatos a medianas

2. // $Y$ acumula as soluções elite como dados para o path-relinking

3. $Y \leftarrow \varnothing$

4. para $\left(k=1, \ldots, M A X k_{\text {PATHRELINKING }}\right)$ faça

5. $\quad / / X_{K}$ recebe, a cada iteração, uma parte (porcentagem)

6. //de vértices como candidatos a medianas, até o total de vértices

7. $X_{K} \leftarrow\left(\frac{k}{M A X k_{\text {PATHRELINKING }}} \times\right.$ DadosORIGINAIS $)$

8. $s \leftarrow s_{k}$

9. $\operatorname{GRASP}\left(X_{K}\right)$

10. $Y \leftarrow Y \cup s_{k}$

11. fim para

12. $\operatorname{GRASP}(Y)$

13. $S_{\text {PATHRELINKING }} \leftarrow s$

14. Retornas $S_{\text {PATHRELINKING }}$

15. fim $\operatorname{GRASP}(Y)$ 


\subsection{A integração do path-relinking com a GRASP}

Considera-se a seguinte meta-heurística híbrida que integra o conceito de path-relinking com a GRASP. A GRASP mantém um conjunto de soluções elite encontradas durante as iterações da meta-heurística. A cada iteração da GRASP, o path-relinking é aplicado, a partir da nova solução gerada pela GRASP, a uma dada solução dentre as soluções elite. A solução alvo pode ser escolhida aleatoriamente a partir do conjunto elite. Como experiência advinda da literatura, pode-se afirmar que os papéis da solução inicial e da solução guia são intercambiáveis. Cada solução pode ser induzida a possibilitar movimentos simultâneos na direção da outra como forma de gerar combinações. Mais detalhes em Laguna \& Martí (1999) e Talbi (2009).

\subsection{0 enfoque de path-relinking sugerido e adotado neste trabalho}

O procedimento de path-relinking construído para este trabalho será descrito a seguir com destaque para novidade que ele introduz em função das características da GRASP e do problema de localização em que ele foi aplicado. Em sequência, uma aplicação foi feita através do ato de simular a formação de clusters com as médias e grandes cidades brasileiras, produzindo um ensaio sobre como utilizar esses recursos na solução de problemas de localização de $p$-medianas.

A primeira providência foi criar um conjunto de soluções elite através de um conceito que aqui foi denominado de "vocação mediana". Este conceito, que será detalhado no decorrer desta descrição, fundamenta-se em um índice - "índice $v m$ " que estabelece o quanto cada cidade, suposta candidata a mediana, está próxima das demais cidades do grupo. Antes de construir esse índice, o instrumento previamente utilizado foi soma das distâncias de cada cidade às demais. Posteriormente, as cidades, supostas candidatas a mediana, foram ordenadas, em ordem crescente, de acordo com a distância total de cada uma delas às demais. Vários problemas menores foram construídos e resolvidos, considerando somente uma porcentagem do total de candidatas a medianas. Diga-se, $\beta \% \times($ Total de candidatas a mediana), para o primeiro problema, $2 \beta \% \times($ Total de candidatas a mediana para o segundo problema), e assim por diante, até que $k \beta \%$ se tornasse 
igual a $100 \%$, isto é, todos os supostos candidatos a medianas. Cuidado foi tomado para que em nenhum dos problemas menores a quantidade de medianas candidatas ficasse inferior ao total máximo de medianas que se desejava localizar. A solução de todos esses problemas menores produziu um aglomerado de medianas, do qual participaram todas as medianas escolhidas como solução dos problemas menores. Esse aglomerado de medianas foi aceito, empiricamente, como um conjunto de medianas associado às soluções elite do problema de localização. Tal aceitação empírica da condição "elite" das soluções assim obtidas, ficou aguardando que experimentos comprovassem a presença de tal atributo. O passo final foi aplicar a esse conjunto de soluções elite um procedimento de path-relinking que explorou a intensificação e a diversificação do processo de busca. Ou seja, um conjunto de novas soluções foi gerado nas várias iterações da GRASP para produzir a solução final que se desejava melhor do que as soluções encontradas antes da intensificação do path-relinking.

\section{O CÓDIGO COMPUTACIONAL}

$\mathrm{O}$ código computacional foi feito em $\mathrm{C}_{++}$. Foram usados alguns componentes da biblioteca STL, o que permitiu desenvolver um código estável, com alto grau de generalidade e baseado em estruturas de dados bastante testadas.

O código foi projetado e construído com base em uma classe de membros, dados e funções, que permitiram modular as várias operações envolvidas na solução do problema. As informações que alimentavam o código foram constituídas do número $|N|=n$ de pontos, cidades ou vértices envolvidos e que formam o conjunto $N$. Além desse número, os dados continham a matriz $n \times n$ simétrica de distâncias, e o número máximo de medianas que a instância pretendia localizar. Um determinado número de ligações compunha algumas entradas da matriz de distâncias. Posteriormente, as demais entradas foram calculadas usando uma rotina que implementa o algoritmo - de programação dinâmica, detalhes em Nemhauser (1966) - de Floyd-Warshall, para encontrar as distâncias mínimas entre todos os pares de pontos ou vértices que se associam às entradas da matriz.

\section{RESULTADOS}

Revista Produção Online, Florianópolis, SC, v.15, n. 3, p.807-829, jul./set. 2015. 
Para mostrar o funcionamento deste modelo de path-relinking foi feita uma aplicação do problema generalizado de $p$-medianas, o que passa a ser feito através da formação de clusters com as cidades brasileiras, médias e grandes, que aparecem na matriz de distâncias do DNIT.

A Tabela 2 mostra as 112 cidades brasileiras que participaram do experimento ordenadas pelo índice de vacação mediana (índice ${ }_{v m}$ ).

Tabela 2 - Índice de vocação mediana das 112 cidades

\begin{tabular}{|c|c|c|c|c|c|}
\hline \multicolumn{3}{|c|}{ Distância } & \multicolumn{3}{|c|}{ Distância } \\
\hline Cidade & Total & Índice $v m$ & Cidade & Total & Índice $_{v m}$ \\
\hline Ribeirão Preto & 147471 & 1,328 & Barbacena & 342656 & 3,086 \\
\hline Campinas & 150832 & 1,358 & Sobral & 342933 & 3,089 \\
\hline Uberlândia & 150823 & 1,358 & Caldas Novas & 343551 & 3,095 \\
\hline São Paulo & 151522 & 1,365 & Petrópolis & 347597 & 3,313 \\
\hline Belo Horizonte & 153667 & 1,384 & Anápolis & 349736 & 3,150 \\
\hline S. José dos Campos & 154141 & 1,388 & Sta. Maria da Vitória & 350263 & 3,155 \\
\hline Goiânia & 157662 & 1,419 & Volta Redonda & 351591 & 3,167 \\
\hline Brasília & 158872 & 1,413 & Itumbiara & 359269 & 3,236 \\
\hline Santos & 159368 & 1,435 & Franca & 359849 & 3,241 \\
\hline Juiz de Fora & 161184 & 1,452 & Guaratinguetá & 360363 & 3,246 \\
\hline Londrina & 164132 & 1,478 & Poços de Caldas & 361194 & 3,254 \\
\hline Rio de Janeiro & 167323 & 1,507 & Uberaba & 361764 & 3,259 \\
\hline Curitiba & 169685 & 1,528 & Parnaíba & 362049 & 3,261 \\
\hline Vitória & 180256 & 1,623 & Barretos & 362978 & 3.270 \\
\hline Joinville & 181321 & 1,633 & Taubaté & 363215 & 3,272 \\
\hline Campo Grande & 190504 & 1,716 & Limeira & 368502 & 3,319 \\
\hline Florianópolis & 195533 & 1,761 & Araraquara & 370828 & 3,340 \\
\hline Feira de Santana & 207691 & 1,871 & Porto Velho & 371620 & 3,347 \\
\hline Caxias do Sul & 217509 & 1,959 & Rio Verde & 371832 & 3,349 \\
\hline Salvador & 217617 & 1,960 & São Carlos & 372180 & 3,352 \\
\hline Palmas & 218699 & 1,970 & Americana & 373571 & 3,365 \\
\hline Cuiabá & 221652 & 1,996 & Sorocaba & 378166 & 3,406 \\
\hline Porto Alegre & 228249 & 2,056 & Imperatriz & 379122 & 3,415 \\
\hline Aracajú & 232642 & 2,095 & S. José do Rio Preto & 379612 & 3,419 \\
\hline Pelotas & 254483 & 2,292 & Bauru & 382036 & 3,441 \\
\hline Paulo Afonso & 256007 & 2,306 & Araçatuba & 384912 & 3,467 \\
\hline Maceió & 257475 & 2,319 & Marília & 388102 & 3,496 \\
\hline Guaranhuns & 260734 & 2,348 & Ourinhos & 391800 & 3,529 \\
\hline Petrolina & 268474 & 2,418 & Presidente Prudente & 396563 & 3,572 \\
\hline Itaparica & 270544 & 2,437 & Rondonópolis & 410760 & 3,700 \\
\hline Caruaru & 2701607 & 2,446 & Paranaguá & 413393 & 3,724 \\
\hline Teresina & 272819 & 2,457 & Maringá & 415095 & 3,739 \\
\hline Itabuna & 273897 & 2,467 & Ponta Grossa & 416291 & 3,750 \\
\hline Recife & 273953 & 2,468 & Tucuruí & 419672 & 3,780 \\
\hline V. da Conquista & 276382 & 2,489 & Itajaí & 429899 & 3,872 \\
\hline Valença & 280867 & 2,530 & Rio Branco & 429826 & 3,872 \\
\hline João Pessoa & 284830 & 2,566 & Blumenau & 434125 & 3,911 \\
\hline Campina Grande & 285137 & 2,568 & Cascavel & 441668 & 3,978 \\
\hline Juazeiro do Norte & 294508 & 2,653 & Lages & 445211 & 4,010 \\
\hline Porto Seguro & 295473 & 2,661 & Dourados & 448614 & 4.041 \\
\hline São Luís & 297497 & 2,680 & Tubarão & 452019 & 4,072 \\
\hline Picos & 298725 & 2,691 & Ponta Porã & 452165 & 4,073 \\
\hline Natal & 301164 & 2,713 & Foz do Iguaçu & 456646 & 4,113 \\
\hline Barreiras & 301376 & 2,715 & Criciúma & 458262 & 4,128 \\
\hline
\end{tabular}


Tabela 2 - Índice de vocação mediana das 112 cidades

\begin{tabular}{|c|c|c|c|c|c|}
\hline Cidade & $\begin{array}{c}\text { Distância } \\
\text { Total }\end{array}$ & Índice $_{v m}$ & Cidade & $\begin{array}{c}\text { Distância } \\
\text { Total }\end{array}$ & Índice $v m$ \\
\hline Ilhéus & 305398 & 2,751 & Passo Fundo & 464060 & 4,180 \\
\hline Fortaleza & 308267 & 2,777 & Corumbá & 468708 & 4,222 \\
\hline Gov. Valadares & 308359 & 2,778 & Manaus & 470779 & 4,241 \\
\hline Belém & 311650 & 2,807 & Novo Hamburgo & 475959 & 4,287 \\
\hline Montes Claros & 312953 & 2,819 & Sinop & 486850 & 4,386 \\
\hline Ipatinga & 314507 & 2,833 & Santa Maria & 494601 & 4,445 \\
\hline Mossoró & 317272 & 2,858 & Vilhena & 515323 & 4,642 \\
\hline Itabira & 320016 & 2,883 & Bagé & 520224 & 4,686 \\
\hline Cachoeiro de Itap. & 333516 & 3,004 & Uruguaiana & 524785 & 4,727 \\
\hline Patos de Minas & 334335 & 3,012 & S. do Livramento & 531354 & 4,786 \\
\hline São Lourenço & 339087 & 3,054 & Chuí & 535793 & 4,826 \\
\hline Campos dos Goyt. & 341316 & 3,074 & Boa Vista & 556965 & 5,017 \\
\hline
\end{tabular}

Pela Tabela 2, vê-se que a cidade brasileira mais próxima das demais cidades médias e grandes - é Ribeirão Preto-SP, com índice ${ }_{v m}$ igual a 1,328. A mais distante das demais é Boa Vista-RR, com índice $v m$ igual a 5,017.

O índice ${ }_{\mathrm{vm}}$ foi construído de acordo com

$$
\text { índice }_{v m}\left(\text { "cidade") }=\frac{\sum(\text { distâncias da "cidade" às outras "cidades" })}{\text { Número total das cidades } \times 1000}\right.
$$

Por exemplo,

$$
\begin{gathered}
\text { índice }_{v m} \text { ("Manaus") }=\frac{\sum(\text { distânciasde Manausàs outras"cidades") }}{\text { Número total dascidades } \times 1000}=4.241 \\
\text { índice }_{v m(\text { ("São Paulo") }}=\frac{\sum \text { (distâncias de São Paulo às outras "cidades" ) }}{\text { Número total das cidades } \times 1000}=1.365
\end{gathered}
$$

No experimento projetado o $\beta$ foi considerado igual a 10. Isto significa que o primeiro problema menor foi resolvido com $10 \%$ das cidades com menor distância total às demais 111 cidades, como candidatas a mediana. O segundo problema menor foi resolvido com $20 \%$ dessas cidades, e assim por diante, até que $100 \%$ das cidades tornaram-se candidatas a mediana na solução do problema de localização em foco. Portanto, foram resolvidas 9 instâncias menores mais a instância original.

A Tabela 3 mostra um conjunto de 26 cidades candidatas a mediana, definido durante a solução das 10 instâncias, conforme já descrito. A Tabela 4 mostra um resumo das soluções de cada uma das 10 instâncias com 2000 iterações. A melhor solução sem o path-relinking ocorreu na quinta instância, ou seja, 20\% das cidades candidatas a mediana, com distância total igual 68551 , entre as medianas e as 
cidades que a elas se ligam. O resultado com path-relinking - Tabela 6 - teve distância total igual 62393. A diferença entre os dois resultados se torna expressiva se for considerado que os dois passaram por um processo de "otimização no sentido das meta-heurísticas". Em outras palavras, o path-relinking trabalha sobre um resultado já otimizado, ou o tem como referência.

Tabela 3 - 26 cidades elite, candidatas a medianas na fase de path-relinking

\begin{tabular}{llll}
\hline Aracajú-SE & Campinas-SP & Campos Grande-MS & Caxias do Sul-RS \\
Cuiabá-MT & Curitiba-PR & Feira de Santana-BA & Florianópolis-SC \\
Fortaleza-CE & Goiânia-Go & João Pessoa-PB & Juiz de Fora-MG \\
Londrina-PR & Maceió-Al & Natal-RN & Pelotas-RS \\
Porto Alegre-RS & Porto Velho-RO & Ribeirão Preto-SP & Rondonópolis-MT \\
Salvador-BA & Santos-SP & S. J. dos Campos-SP & São Paulo-SP \\
Teresina-PI & Uberlândia-MG & & \\
\hline
\end{tabular}

Tabela 4 - Resumo dos resultados com 112 cidades, 5 medianas, 2000 iterações

\begin{tabular}{cccc}
\hline Instância & Trocas para melhor solução & Distância total & Tempo médio por iteração(seg) \\
\hline 1 & 8 & 108493 & 0,03250000 \\
2 & 9 & 68551 & 0,06450000 \\
3 & 3 & 68613 & 0.09950000 \\
4 & 8 & 68899 & 0,13349999 \\
5 & 7 & 70795 & 0.16850001 \\
6 & 4 & 71048 & 0,20299999 \\
7 & 5 & 71546 & 0,24050000 \\
8 & 2 & 73940 & 0,28650001 \\
9 & 8 & 72485 & 0,33600000 \\
10 & 4 & 75445 & 0,38350001 \\
\hline
\end{tabular}

Experimento semelhante, agora com 20000 iterações para resolver os problemas menores, tem o resultado resumido na Tabela 5. Neste caso, a melhor solução sem o path-relinking é a distância total igual a 66086. Com o path-relinking o valor melhora para 62393. Os valores da distância total sem o path-relinking são diferentes, mostrando que um número maior de iterações influenciou o resultado. Entretanto, a distância total com path-relinking foi o mesmo nos dois casos apresentados. O caso de 20000 iterações para resolver cada um dos problemas menores, gerou uma elite de 22 cidades, diferente do caso anterior, de 2000 iterações, que gerou uma elite de 26 cidades. Nos dois casos as cidades elites foram candidatas a mediana na fase de intensificação do path-relinking.

Com fator de intensificação do path-relinking igual a 10 a solução foi a mesma, e a única diferença foi a ordem em que o algoritmo definiu as medianas com Revista Produção Online, Florianópolis, SC, v.15, n. 3, p.807-829, jul./set. 2015. 
os clusters associados, de acordo com a Tabela 7. Suspeita-se que esta seja uma solução ótima para este número de medianas.

Tabela 5 - Resumo dos resultados com 112 cidades, 5 medianas, 20000 iterações

\begin{tabular}{cccc}
\hline Instância & Trocas para melhor solução & Distância total & Tempo médio por iteração(seg) \\
\hline 1 & 3 & 108493 & 0,03335000 \\
2 & 6 & 68551 & 0,06450000 \\
3 & 9 & 66362 & 0.10200000 \\
4 & 6 & 66086 & 0,14135000 \\
5 & 13 & 68300 & 0,18560000 \\
6 & 10 & 68631 & 0,22485000 \\
7 & 19 & 67489 & 0,27375001 \\
8 & 8 & 67329 & 0,37114999 \\
9 & 14 & 70217 & 0,41319999 \\
10 & 13 & 69277 & 0,47815001 \\
\hline
\end{tabular}

Tabela 6 - Resultado com path-relinking

(continua)

DISTÂNCIA TOTAL DA MELHOR SOLUÇÃO ENCONTRADA: 62393

1) Cluster da mediana FEIRA DE SANTANA-BA:

$\begin{array}{lll}\text { ARACAJÚ-SE } & \text { BARREIRAS-BA } & \text { CAMPINA GRANDE-PB } \\ \text { GUARANHUNS-PE } & \text { ILHÉUS-BA } & \text { ITABUNA-BA } \\ \text { JOÃO PESSOA-PB } & \text { MACEIÓ-AL } & \text { NATAL-RN } \\ \text { PETROLINA-PE } & \text { PORTO SEGURO-BA } & \text { RECIFE-PE } \\ \text { S. Ma DA VITÓRIA-BA } & \text { VALENÇA-BA } & \text { V. DA CONQUISTA-BA } \\ \text { Tatal } & \end{array}$

Total de cidades neste cluster: 19. Distância (custo): 10557

2) Cluster da mediana RIBEIRÃO PRETO-SP:

\begin{tabular}{|c|c|c|c|}
\hline MERICANA-SP & ANÁPOLIS-GO & ARAÇATUBA-SP & ARARAQUARA-SP \\
\hline BARRETOS-SP & BARBACENA-MG & BAURU-SP & B. HORIZONTE-MG \\
\hline BRASÍLIA-DF & C. DE ITAPEMIRIM-ES & CALDAS NOVAS-GO & CAMPINAS-SP \\
\hline MPO GRANDE-MS & CAMPOS DOS GOYT-RJ & CASCAVEL-PR & CORUMBÁ-MS \\
\hline CUIABÁ-MT & CURITIBA-PR & DOURADOS-MS & F. DO IGUAÇÚ-PR \\
\hline ANCA-SP & COIÂNIA-GO & GOV. VALADARES-MG & GUARATINGUETÁ-SP \\
\hline IPATINGA-MG & ITABIRA-MG & ITUMBIARA-GO & JUIZ DE FORA-MG \\
\hline IMFIRA_SP & LONDRINA-PR & MARÍLIA-SP & MARINGÁ-PR \\
\hline MONTES CLAROS-MG & OURINHOS-SP & PARANAGUÁ-PR & PATOS DE MINAS-MG \\
\hline 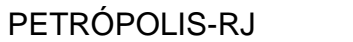 & POCOS DE C.-MG & PONTA GROSSA-PR & PONTA PORÂ-MS \\
\hline PRES. PRUDENTE-SP & RIO DE JANEIRO-RJ & RIO VERDE-GO & RONDONÓPOLIS-MT \\
\hline & ARLOS-SP & S. J. DO RIO PRETO-SP & S. J. DOS CAMPOS-SP \\
\hline IO LOUREN & SÃO PAULO-SP & SINOP-MT & SOROCABA-SP \\
\hline TAUBATÉ-S & UBERABA-MG & UBERLÂNDIA-MG & VITÓRIA-ES \\
\hline
\end{tabular}

VOLTA REDONDA-RJ

Total de cidades neste cluster: 57. Distância (custo) total neste cluster: 33771

3) Cluster da mediana PORTO VELHO-RO:

BOA VISTA-RR MANAUS-AM

Total de cidades neste cluster: 4. Distância (custo) total neste cluster: 3835

ITAPARICA-PE

PAULO-AFONSO-BA

SALVADOR-BA

RECIFE-PE

4) Cluster da mediana PORTO ALEGRE-RS:

BAGÉ-RS BLUMENAU-SC

CRICIÚMA-SC

FLORIANÓPOLIS-SC

CAXIAS DO SUL-RS ITAJAÍ-SC

CHUÍ-RS

JOINVILLE-SC 
Tabela 6 - Resultado com path-relinking

(conclusão)

DISTÂNCIA TOTAL DA MELHOR SOLUÇÃO ENCONTRADA: 62393

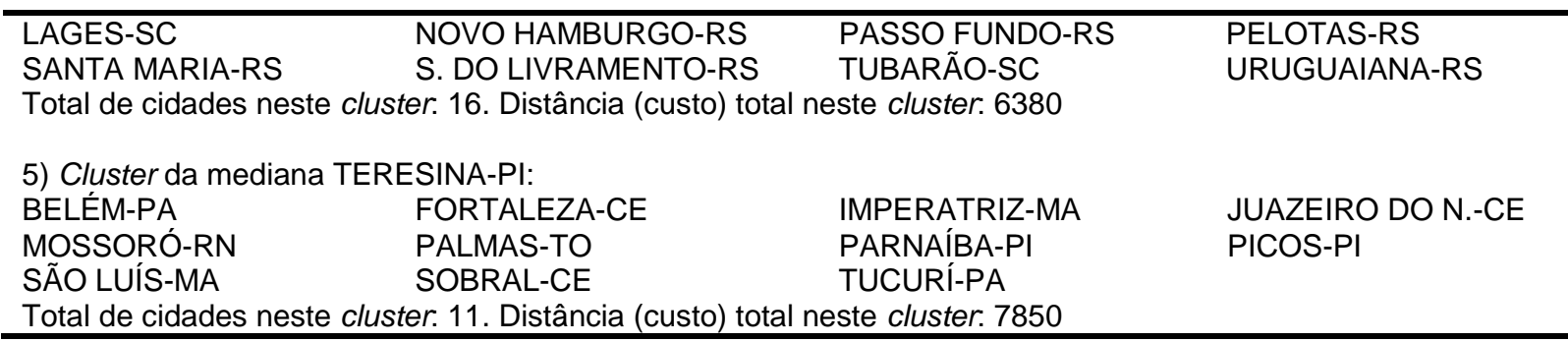

Tabela 7- Resultado com path-relinking após 200000 iterações, e 20000 iterações na solução de cada um dos problemas menores

\section{DISTÂNCIA TOTAL DA MELHOR SOLUÇÃO ENCONTRADA: 62393}

Total de cidades neste cluster: 19. Distância (custo): 10557

1) Cluster da mediana RIBEIRÃO PRETO-SP:

\begin{tabular}{lll} 
AMERICANA-SP & ANÁPOLIS-GO & ARAÇATUBA-SP \\
BARRETOS-SP & BARBACENA-MG & BAURU-SP \\
BRASÍLIA-DF & C. DE ITAPEMIRIM-ES & CALDAS NOVAS-GO \\
CAMPO GRANDE-MS & CAMPOS DOS GOYT-RJ & CASCAVEL-PR \\
CUIABÁ-MT & CURITIBA-PR & DOURADOS-MS \\
FRANCA-SP & COIÂNIA-GO & GOV. VALADARES-MG \\
IPATINGA-MG & ITABIRA-MG & ITUMBIARA-GO \\
LIMEIRA-SP & LONDRINA-PR & MARÍLIA-SP \\
MONTES CLAROS-MG & OURINHOS-SP & PARANAGUÁ-PR \\
PETRÓPOLIS-RJ & POÇOS DE C.-MG & PONTA GROSSA-PR \\
PRES. PRUDENTE-SP & RIO DE JANEIRO-RJ & RIO VERDE-GO \\
SANTOS-SP & SÃO CARLOS-SP & S. J. DO RIO PRETO-SP \\
SÃO LOURENÇO-MG & SÃO PAULO-SP & SINOP-MT \\
TAUBATÉ-SP & UBERABA-MG & UBERLÂNDIA-MG \\
\hline
\end{tabular}

ARARAQUARA-SP

B. HORIZONTE-MG

CAMPINAS-SP

CORUMBÁ-MS

F. DO IGUAÇÚ-PR

GUARATINGUETÁ-SP

JUIZ DE FORA-MG

MARINGÁ-PR

PATOS DE MINAS-MG

PONTA PORÃ-MS

RONDONÓPOLIS-MT

S. J. DOS CAMPOS-SP

SOROCABA-SP

VITÓRIA-ES

VOLTA REDONDA-RJ

Total de cidades neste cluster: 57. Distância (custo) total neste cluster: 33771

2) Cluster da mediana PORTO VELHO-RO:

BOA VISTA-RR MANAUS-AM

Total de cidades neste cluster: 4. Distância (custo) total neste cluster: 3835

RIO BRANCO-AC VILHENA-RO

3) Cluster da mediana PORTO ALEGRE-RS:

BAGÉ-RS BLUMENAU-SC

CRICIÚMA-SC

LAGES-SC

SANTA MARIA-RS

FLORIANÓPOLIS-SC

NOVO HAMBURGO-RS

S. DO LIVRAMENTO-RS

CAXIAS DO SUL-RS

ITAJAÍ-SC

PASSO FUNDO-RS

Total de cidades neste cluster: 16. Distância (custo) total neste cluster: 6380

4) Cluster da mediana TERESINA-PI:

$\begin{array}{lll}\text { BELÉM-PA } & \text { FORTALEZA-CE } & \text { IMPERATRIZ-MA } \\ \text { MOSSORÓ-RN } & \text { PALMAS-TO } & \text { PARNAÍBA-PI } \\ \text { SÃO LUÍS-MA } & \text { SOBRAL-CE } & \text { TUCURÍ-PA }\end{array}$

Total de cidades neste cluster: 11. Distância (custo) total neste cluster: 7850

5) Cluster da mediana FEIRA DE SANATANA-BA:
ARACAJÚ-SE
BARREIRAS-BA
GUARANHUNS-PE
ILHÉUS-BA
MACEIÓ-AL
PORTO SEGURO-BA
PETROLINA-PE
VALENÇA-BA
CAMPINA GRANDE-PB
ITABUNA-BA
NATAL-RN
RECIFE-PE
V. DA CONQUISTA-BA
S. Mํㅡㄹ DA VITÓRIA-BA

Total de cidades neste cluster: 19. Distância (custo) total neste cluster: 10557

\author{
CHUÍ-RS \\ JOINVILLE-SC \\ PELOTAS-RS \\ URUGUAIANA-RS
}

JUAZEIRO DO N.-CE PICOS-PI
ITAPARICA-PE

PAULO-AFONSO-BA

SALVADOR-BA 
Um fato que chama atenção é o de Ribeirão Preto-SP, cidade brasileira com melhor índice de vocação mediana, ser a mediana do maior cluster. A Tabela 8 mostra uma possível correlação entre o tamanho do cluster e o índice de vocação mediana da cidade em questão: na medida em que piora o índice ${ }_{v m}$, diminui $o$ tamanho do cluster atendido por aquela mediana. Esta possível correlação entre o tamanho do cluster e o índice $v m$ não foi estudada neste trabalho.

Tabela 8 - Quando piora o índice de vocação mediana, diminui o tamanho do cluster

\begin{tabular}{lcc}
\hline \multicolumn{1}{c}{ Mediana } & Número de cidades no cluster & Índice vocação mediana \\
\hline Ribeirão Preto-SP & 57 & 1,328 \\
Feira de Santana-BA & 19 & 1,871 \\
Porto Alegre-RS & 16 & 2,056 \\
Teresina-PI & 11 & 2,454 \\
Porto Velho-RO & 4 & 3,347 \\
\hline
\end{tabular}

\section{CONCLUSÕES}

Este trabalho se baseou na hipótese de que havia espaço para aprimoramento de métodos que resolvem problemas de otimização usando metaheurísticas. Em particular, a escolha para demonstrar a veracidade desta hipótese recaiu sobre a meta-heurística GRASP, seus métodos reativos e procedimentos de path-relinking, especializados na solução do modelo generalizado de localização de p-medianas. Para alcançar o objetivo buscou-se explorar o processo reativo da GRASP por duas vias, ou seja, adotando o que se passou a denominar de "GRASP duplamente reativa", já que dois parâmetros de reação passaram a atuar, cooperativamente, nesse cenário. Para entendimento próprio e para comunicar o procedimento à comunidade interessada no assunto, passou-se a explicar tal procedimento com uma metáfora que aqui foi denominada de "metáfora da peneira". Todo o esforço feito confirmou a boa reputação da meta-heurística GRASP no contexto da resolução de problemas combinatórios destinados a aplicações tecnológicas do cotidiano.

A questão básica aqui tratada se constitui de como decidir, a partir de locais elegíveis conhecidos ou a partir de um grande número de locais descritos como área, uma localização para instalar uma facilidade e como atribuir ou alocar clientes a ela.

O passo seguinte foi o de explorar as capacidades do path-relinking. As experiências realizadas mostraram tratar-se de um procedimento capaz de Revista Produção Online, Florianópolis, SC, v.15, n. 3, p.807-829, jul./set. 2015. 
aproximar os resultados da GRASP aos de soluções dos algoritmos exatos. A principal proposta desse método é a de intensificar a busca entre soluções elite encontradas nas fases anteriores do algoritmo. De todas as formas acredita-se que não está esgotado o espaço de aprimoramentos aqui explorado, o que constitui estímulo para prosseguir testando ideias semelhantes. Além disso, tal esforço sempre se ocupa de um aspecto particular desse problema que é amplo e variado nas diversas modalidades do problema e suas aplicações.

As simulações realizadas passaram a ser conduzidas com dados reais, se enquadrando no que se pode chamar de contribuição à logística de localização. A extensão dos experimentos foi relativamente grande na medida em que se procurou variar a quantidade de clusters, o número de iterações, assim como os valores do parâmetro de intensificação do path-relinking que passou a ser usado em caráter definitivo.

Um subproduto interessante dessa fase de simulação de clusters foi a invenção de um índice que passou a ser chamado de "índice de vocação mediana" que é uma representação numérica capaz de medir a proximidade da cidade em questão com as demais cidades brasileiras, médias e grandes, constantes da matriz do DNIT. Esta é uma informação logística de valor tanto para um estudo locacional qualitativo, quanto para projetos de localização em que se explora o lado quantitativo. A pesquisa descobriu por via dos experimentos conduzidos que a cidade detentora do melhor índice de vocação mediana é RIBEIRÃO PRETO-SP, e a cidade com o pior índice é BOA VISTA-RR. Essa informação não exclui as cidades intermediárias em relação a este índice, já que participando de uma análise locacional elas podem apresentar outras características interessantes, tanto no aspecto qualitativo, quanto do lado quantitativo.

\section{REFERÊNCIAS}

AIEX, R. M.; BINATO, S.; RESENDE, M. G. G. Parallel GRASP with path-relinking for job shop scheduling. Parallel Computing - Special issue: Parallel computing in numerical optimization, v. 29, n.4, p. $393-430,2003$. http://dx.doi.org/10.1016/S0167-8191(03)00014-0

ALVIM, A.; RIBEIRO, C. Load balancing in the parallelization of the metaheuristic GRASP. Technical report, Department of Computer Science, Catholic University of Rio de Janeiro, Rio de Janeiro, Brazil.1988.

BATTITI, R. Reactive search: towards self-tuning heuristics. In V. J. Rayward- Smith, I. H. Osman, C. R. Reeves, G. D. Smith, editors. Modern Heuristic Search Methods. 1996. Revista Produção Online, Florianópolis, SC, v.15, n. 3, p.807-829, jul./set. 2015. 
BERMAN, O.; DREZNER, Z. A note on the location of an obnoxious facility on a network. European Journal of Operational Research, n. 120, p. 215-217, 2000. http://dx.doi.org/10.1016/S0377-2217(98)00237-9

BOUDIA, M.; LOULY, M.A.O., e PRINS, C. A reactive GRASP and path-relinking for a combined production-distribution problem. Computers \& Operations Research, n. 34, p. 3402 - 3419, 2007. http://dx.doi.org/10.1016/j.cor.2006.02.005

CAIRES, L. F. V.; ASSIS, L. P. e, ANDRADE, A. V. Aplicação do método path-relinking na resolução do problema roteamento de veículos com coleta e entrega simultâneas. In: XLII SBPO. 31, 38, 2010, Bento Gonçalves. Anais... Bento Gonçalves: SOBRAPO, 2010, p. $1745-1756$.

FEO, T. A.; RESENDE, M. G. C. Greedy randomized adaptive search procedures. Journal of Global Optimization, 6, p. 109 - 133, 1995. http://dx.doi.org/10.1007/BF01096763

FRINHANI, R. M. D. GRASP com path-relinking para agrupamento de dados biológicos. Originalmente apresentada como dissertação de mestrado, Instituto de Ciências Exatas Universidade Federal de Minas Gerais, 2011.

http://hdl.handle.net/1843/SLSS-8GQJ46

GARCÍA, J. A. D. Algorithmic approaches for the single source capacitated plant location problem. Originalmente apresentada como tese de doutorado, Universitat Politécnica de Catalunya, 2001.

GLOVER, F., LAGUNA, M., \& MARTÍ, R. Scatter search and path-relinking: Advances and applications. $30-55,2003$.

http://dx.doi.org/10.1007/0-306-48056-5 1

GLOVER, F.; KOCHENBERGER, G. A.. Handbook of Metaheuristics. International series in operations research \& management science. Boston, Dordrecht, London: Kluwer Academic Publishers. 55. Eds 2003.

http://dx.doi.org/10.1007/b101874

LAGUNA, M. \& MARTÍ, R. GRASP and path-relinking for 2-layer straight line crossing minimization. INFORMS J. on Computing, v. 11, n. 1, p. 44-52. 57, 1999.

http://pubsonline.informs.org/doi/pdf/10.1287/ijoc.11.1.44

MARTINS, S.; RESENDE, M.; RIBEIRO, C. e PARDALOS, P. A parallel GRASP for the Steiner tree problem in graphs using a hybrid local search strategy. Journal of Global Optimization, 17, p. 267-283, 2000. http://dx.doi.org/10.1023/A:1026546708757

NEMHAUSER, G. L. Introduction to Dynamic Programming. Series in decision and control. Wiley. 69, 1966.

RANGEL, M. C., ABREU, N. M. M., \& NETTO, P. O. B. GRASP para o pqa: um limite de aceitações para soluções iniciais. Pesquisa Operacional, v. 20, n. 1, p. 46-58. 32, 47. 2000. http://dx.doi.org/10.1590/S0101-74382000000100006

RESENDE, M. G. C.; WERNECK, R. F. A GRASP with path-relinking for the p-median problem. AT\&T Labs Research Technical Report TD-5E53XL, 2002.

http://citeseerx.ist.psu.edu/viewdoc/summary?doi=10.1.1.19.9495 
TALBI, E.G. Metaheuristics: From Design to Implementation. John Wiley \& Sons, 2009. http://dx.doi.org/10.1002/9780470496916

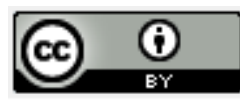

Artigo recebido em 27/06/2013 e aceito para publicação em 25/06/2015

DOI: http://dx.doi.org/ 10.14488/1676-1901.v15i3.1628 